\title{
Análisis de los factores de la deserción estudiantil en una facultad de las unidades tecnológicas de Santander, Bucaramanga, Colombia
}

\author{
Cristian E.Peña Torres* \\ Universidad de Granada \\ Tomas Sola Martinez** \\ Universidad de Granada \\ Juan Antonio Lopez Nuñez ${ }^{* * *}$ \\ Universidad de Granada
}

\begin{abstract}
Resumen: Las Unidades Tecnológicas de Santander, es una institución de Educación Superior, líder local dentro de las de su misma naturaleza, e igual que ellas presenta altos niveles de deserción, factor prioritario dentro de los estudios a nivel nacional y que se ha convertido en requisito de verificación en procesos de acreditación, de implementación obligatoria. Las estadísticas muestran niveles de deserción que superan el $50 \%$. De esta situación surge la inquietud de cuáles son las causas que generan el abandono de los programas de educación superior y las posibles alternativas para aumentar los niveles de retención. Partiendo de referentes teóricos que abordan el fenómeno de la deserción, el proceso de investigación siguió los lineamientos de una metodología mixta de corte descriptivo. Los instrumentos de recogida de datos fueron de tipo cualitativo y cuantitativo, permitiendo obtener resultados válidos esperados; que en primer momento fueron los datos sistematizados para establecer y priorizar las causas de la deserción; en segundo momento el análisis de los datos a través del cruce la información; y en tercer lugar proposición de estrategias que contribuyan a fijar metas y políticas institucionales.

Palabras clave: Educación Superior; Fracaso Académico; Prevención de la deserción académica, Permanencia.

Abstract: The Technological Units of Santander, it is an institution of Higher Education. This is local leader within of the same natural, and like them present high levels of desertion, priority factor in studies nationwide and that has become verification requirement accreditation processes, which at this time they are mandatory implementation. Statistics show dropout rates exceeding 50\%. In this situation the concern arises of what are the causes of the desertion of higher education programs and possible alternatives to increase retention levels. Based on theoretical references that address the phenomenon of desertion from various theories and approaches, the reachersproccess fallowed the guidelines of a mixed methodology cutting descriptive. For this reason the data collection instruments were qualitative and quantitative, allowing obtain valid results expected; that the first time data were systematized to establish and prioritize the causes of desertion: in the second time date analysis through the crossing information; and thirdly proposing strategies to help set goals and institutional policies.
\end{abstract}

Keywords: higher education, Failure academic, prevention of academic attrition, Permanence.

\section{Introducción}

La Revolución Educativa en Colombia, se enmarca en tres criterios: Cobertura, Calidad y Eficiencia. Entendidos como: la posibilidad de llegar a más

\footnotetext{
* Psicólogo, Universidad Pontificia Bolivariana, Bucaramanga, Colombia. Doctorando en Currículo, profesorado e instituciones educativas, Universidad de Granada, Granada, España. Profesor y coordinador del grupo de permanencia y graduación de las Unidades Tecnológicas de Santander. Correo cristianpsicologo@yahoo.es

** Licenciado en Pedagogía y Psicología, Universidad de Granada, España. Doctor en Filosofía y Ciencias de la Educación, UNAD, Madrid. Profesor Titular de la Universidad de Granada. Correo electrónico: tsola@ugr.es

*** Doctor en Ciencias de la Educación, Universidad de Granada, Granada, España. Profesor, Departamento de Didáctica y Organización Escolar, Universidad de Granada, Granada, España. Correo electrónico: juanlope@ugr.es
} 
personas; las condiciones en que debe darse el servicio y el logro de los fines propuestos. En este sentido la Cobertura en Educación superior no se agota con que el estudiante se matricule en un programa, sino que culmine su formación profesional para poder cumplir el criterio de eficiencia.

La retención de un estudiante hasta culminar el programa profesional, se convierte en el fin de las instituciones de educación superior y en criterio de validez para calificar la calidad, es por ello que los niveles de deserción son entendidos como fracaso, no solo del estudiante sino de la institución que no logra la graduación de todos los estudiantes.

El presente trabajo agota la primera etapa de un camino orientado a disminuir la deserción, su fin principal es establecer los factores que influyen y servir de punto de partida para un plan de mejoramiento y de prevención de la deserción.

Para el logro de los objetivos se siguieron herramientas investigativas de tipo cualitativo y cuantitativo, partiendo de la construcción de un instrumento tipo cuestionario, aplicado a desertores; corroborando la información obtenida con entrevistas, grupo focal y revisión documental. Los instrumentos de tipo cuantitativo se sometieron a análisis estadístico para validarlo y extraer las conclusiones.

El objetivo general se encaminó a diagnosticar las causas de deserción estudiantil en los programas académicos de las UTS y proponer alternativas de retención estudiantil.

Los objetivos específicos se centraron en establecer la tendencia, de los factores que influyen en la deserción; realizar un comparativo entre la prueba de acceso SABER 11 (prueba estatal generalizada que evalúa las competencias para acceder a la educación superior) y los resultados académicos obtenidos en el primer semestre; Determinar estadísticamente el grado de incidencia que tienen los costos educativos, el estrato social, los factores académicos y los factores personales y familiares, frente a la decisión de abandonar un programa de estudio.

La muestra estuvo conformada por 371 desertores de los diferentes programas de la facultadde Ciencias Socioeconómicas y Empresariales, donde se encontraban matriculados 8.590 estudiantes en el ciclo tecnológico, como el estudio no discrimina por género, semestre, carrera, estrato socioeconómico ni otros factores sociodemográficos, la población de la facultad se considera homogénea y por tanto la muestra es calculada mediante un muestreo aleatorio simple.

La recogida de datos se llevó a cabo utilizando instrumentos, que por su naturaleza y aplicabilidad, permitieran el cruce de la información y su contrastación, en consonancia con la metodología mixta que desarrolla la presente investigación. En ese sentido se diseñó y valido el cuestionario, aplicándolo a la muestra seleccionada, se aplicaron entrevistas y se realizó un grupo focal; de la triangulación de la información se obtuvieron las conclusiones, avaladas por el análisis estadístico.

A partir de la validación, ejecución y tabulación del instrumento se realiza una reducción factorial a cada una de las categorías con escala Likert (categorías de la 4 a la 8) definidas durante la investigación: Actitudinal y vocacional (4), socioeconómicas (5), Personales y familiares (6), Institucionales (7) y Hábitos y métodos de estudio (8). Con el fin de analizar si existe más de un tipo de agrupamiento.

Estas son analizadas mediante dos estadísticos con el fin de garantizar la consistencia interna de las preguntas (a partir de su correlación Campo-Arias y Oviedo, (2008), Pardo Merino y Ruiz Diaz, (2002), la Medida Kaiser Meyer Olkin (KMO)y la esfericidad de Barlett EB. 
Una vez validadas las categorías y definidas las preguntas que conforman cada factor se analiza su nivel de incidencia mediante la tendencia factorial, es decir, para todas las preguntas agrupadas en su respectivo factor se realiza un diagrama de frecuencias acumuladas con el fin de determinar cuál fue la tendencia poblacional según la escala definida en el instrumento: Nunca ó no, Algunas veces, Casi siempre, Siempre ó si, para este caso particular.

Finalmente, se seleccionan aquellos factores que influyeron en el nivel de deserción y se agrupan por subcategoría, lo anterior con el fin de determinar futuras acciones para disminuir dicho indicador.

La información y conclusiones obtenidas a través de este trabajo servirán de insumo a la institución para el plan de mejora permanente, además de la caracterización de ciertos aspectos que influyen en el fenómeno de la deserción y sus distintas variables.

\section{Antecedentes}

Vielka De Escobar (2005:15) precisa que "deserción es el proceso de abandono, voluntario o forzado de la carrera en la que se matricula un estudiante, por la influencia positiva o negativa de circunstancias internas o externas a él o ella". En lo que respecta a la educación superior entre uno y otro periodo académico (semestre o año). (De Escobar, 2005)

En el glosario de la Red Iberoamericana para la acreditación de la calidad de la Educación Superior, se considera la deserción como un sinónimo de abandono, denominado también mortalidad escolar que se define como estudiantes que suspenden, repiten, cambian de carrera, o abandonan antes de obtener el título. (Glosario Riaces,2004).

De acuerdo a un estudio patrocinado por la Asociación Nacional de Universidades e Instituciones de Educación Superior, la deserción es entendida como la suspensión definitiva o temporal, voluntaria o forzada; distinguiéndose diferentes modalidades de deserción, como: abandono de la carrera, abandono de la institución y abandono del sistema de educación superior; dentro de las suspensiones también se contempla el cambio de carrera o programa. (ROMO, 2007).

Al conjugar las definiciones de deserción dadas por Tinto (1982) y Giovagnoli (2002), se puede entender la deserción como una situación a la que se enfrenta un estudiante cuando aspira y no logra concluir su proyecto educativo, considerándose como desertor a aquel individuo que siendo estudiante de una Institución de Educación Superior no presenta actividad académica durante dos semestres académicos consecutivos, lo cual equivale a un año de inactividad académica.

En cuanto a los determinantes de la deserción, Álvarez, (1997:49), citando a Baquero, asocia a cuatro factores las causas principales de la deserción:

a) Factores personales: constituidos por motivos psicológicos, que comprenden aspectos motivacionales, emocionales, desadaptación e insatisfacción de expectativas; motivos sociológicos, debidos a influencias familiares y de otros grupos como amigos, condiscípulos, vecinos; y otros motivos no clasificados como la edad, salud, fallecimiento, entre otros.De igual forma los factores de grupo muestran un impacto en la calidad de las relaciones interpersonales, por ejemplo, la cultura de trabajo, las redes y el grado de apoyo institucional recibido de responsables académicos. (ION, 2014)

b) Factores académicos: dados por problemas cognitivos como bajo rendimiento académico, repitencia, ausencia de disciplina y métodos de estudio; 
deficiencias universitarias como dificultades en los programas académicos que tienen que ver con la enseñanza tradicional, insatisfacción académica generada por la falta de espacios pedagógicos adecuados para el estudio, falta de orientación profesional que se manifiesta en una elección inadecuada de carrera o institución y ausencia de aptitud académica.

c) Factores socio-económicos: generados por bajos ingresos familiares, desempleo, falta de apoyo familiar, incompatibilidad de horario entre trabajo y estudio.

d) Factores Institucionales: causados por el cambio de institución, deficiencia administrativa, influencia negativa de los docentes y programas académicos obsoletos y rígidos, baja calidad educativa. (AMAYAet al., 2016).

Sobre la influencia de estos factores en el éxito de la educación superior se afirma que todo profesor ha de complementar su labor docente con su acción orientadora y tutorial, ejerciéndola simultáneamente (ORTIZ, 2005). Más aún se considera que la función tutorial debe trascender a todos los ámbitos relacionados con el alumno universitario, no puede limitarse a la transmisión del conocimiento a través de la cátedra. En consecuencia, la acción tutorial del maestro ha de favorecer el trabajo individual y el trabajo en equipo, contribuyendo con ello a aumentar las posibilidades de permanencia.

El informe presentado por el Ministerio de Educación sobre la educación superior en Colombia, ratifica estos factores asociándolos a teóricos de gran trayectoria en el tema, como se observa en el siguiente esquema:

Fuente: (CASTAÑO et al., 2008)

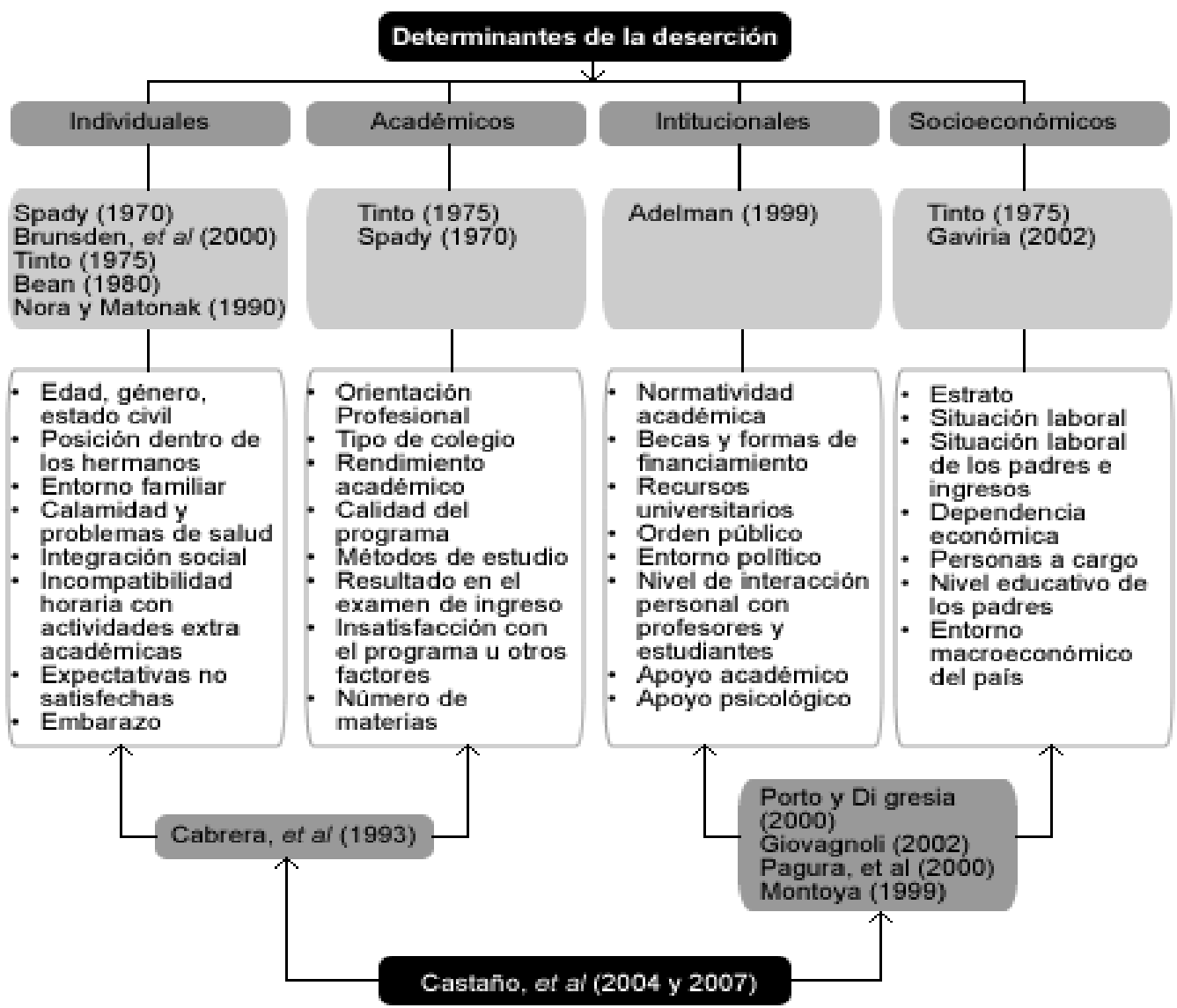

Figura 1. Factores determinantes de la deserción 
Autores como Cabrera; Castañeda; y Nora. (1992) han realizado estudios que compendian gran parte de la teoría existente, permitiendo clasificar enfoques y modelos desde distintas perspectivas. Otros teóricos han explicado el fenómeno de la deserción a través de modelos. (CABRERA el al., 2006) es así como ellos identifican cuatro modelos, en línea coherente con los enfoques:

Modelo de adaptación: la deserción se ocasiona por una adaptación e integración insuficiente del estudiante en el ambiente escolar y social de la enseñanza universitaria. Se fundamenta en el concepto de anomia de Durkheim (Girola, 2005), que lo empleaba para describir la falta de integración del individuo en el contexto (social, económico, cultural u organizacional). En la anomia el individuo no reconoce las normas que rigen los grupos sociales y por consiguiente no se integra y se excluye del contexto.

Dentro de este modelo de adaptación hay que destacar la Teoría de la Persistencia de Vicent Tinto, $(1975,1989,1993)$ quien atribuye la permanencia en la educación superior, al grado de ajuste entre el estudiante y la institución, adquirido a partir de las experiencias académicas y sociales (integración) y esta integración depende de: las experiencias durante la permanencia en la universidad, las experiencias previas al acceso universitario y las características individuales, que, por otro lado, son susceptibles a las políticas y prácticas universitarias. Estudios posteriores Pascarella y Terenzini(1991) ratificaron este impacto de la integración académica sobre la persistencia.

Otra teoría integrada en este modelo adaptativo es la del Agotamiento Estudiantil (Attrition) de Bean y Metzner(1985), que pretende explicar el proceso de agotamiento en los estudiantes universitarios no tradicionales, definidos como aquellos de más de 24 años de edad, que no dependen de sus padres para su sostenimiento, que asisten a la universidad a tiempo parcial, o alguna combinación de esas tres situaciones.

Nora (2002) analiza las interrelaciones que se producen entre los "ritos de transición" que recoge el modelo de integración estudiantil de Tinto (1993), y el apoyo que los estudiantes reciben de los "otros significativos", contemplados en el modelo de adaptación estudiantil de Nora y Cabrera (1996). Según esta autora, de esta interrelación surgen una serie de factores que tienen una incidencia directa en: 1) la integración y las experiencias sociales y académicas; 2) los niveles de compromiso para alcanzar las metas escolares e institucionales; y 3) la decisión para abandonar o permanecer implicado en los estudios universitarios.

Relacionada también con el modelo de adaptación, Holland (1966), establece una correspondencia entre tipos de personalidad y adaptación al ambiente (RIASEC = Realista, Investigador, Artístico, Social, Emprendedor y Convencional). Para Holland, las posibilidades de adaptación y éxito personal están en función de las características de personalidad de cada estudiante, de tal manera que en función de dichos rasgos habrá ambientes más o menos propicios para la adaptación. En esta línea, los trabajos de Seligman (1990) sobre el optimismo aprendido, sugieren que el estudiante optimista opera con una visión positiva sobre sus capacidades y recursos influyendo sobre las condiciones del ambiente, para optimizarlo y encontrar las oportunidades situacionales que le permitan la consecución del éxito.

Modelo estructural: la deserción es el resultado de las contradicciones de los diferentes subsistemas (político, económico y social) que integran el sistema social en su conjunto, y que finalmente influyen en las decisiones del alumnado de abandonar el 
programa académico elegido. A modo de ejemplo, Thomas (2002), partiendo del concepto de "hábito institucional" de Bourdieu y Passeron (1977), sugieren que la institución universitaria tiende a reproducir las normas y hábitos de un grupo social particular, limitando las posibilidades a los estudiantes de distinta procedencia social.

Desde esta perspectiva se entiende que el abandono es un fenómeno inherente al sistema social, por lo que es difícil aislar los motivos específicos y reales que conducen a los estudiantes a la deserción (Luján y Resendiz, 1981).

Modelo economicista: afirma que las inversiones en educación superior se alejan cada vez más de los objetivos universitarios, necesitándose la rápida búsqueda de autofinanciación. Se puede afirmar que la gestión universitaria, se direcciona hacia el rendimiento económico, utilizando la producción del conocimiento como un medio y no como un fin en sí mismo (Zabalza, 2002).

Modelo psicopedagógico: Se centra principalmente en aportes de Cabrera, L., Tomás, J., Álvarez, P. y González, M. (2006), desarrollado a partir de diferentes teorías, trabajos empíricos y aportes propios. La esencia de este modelo está constituida por aspectos de los modelos de adaptación y estructural, más otras dimensiones de carácter psicoeducativo no incluidas por éstos. Al analizar factores personales, institucionales y sociales en estudiantes que abandonan sus estudios, se encuentra que las variables psicológicas y educativas son las que más determinan su éxito o fracaso. Aseguran los autores citados que la revisión de la literatura permite encontrar investigaciones previas que vienen confirmando la relación existente entre la decisión de abandonar y las variables de carácter psicopedagógico.

Como ilustración de los trabajos que sirvieron de fundamento al modelo psicopedagógico, se encuentran investigaciones como la de Ryan y Glenn (2002) quienes demuestran la eficacia de la instrucción en estrategias de aprendizaje para obtener un incremento en las tasas de retención del alumnado universitario de nuevo ingreso. Wasserman (2001) encuentra diferencias significativas en variables psicológicas y evolutivas entre los universitarios que abandonan sus estudios y los que continúan. Asimismo, Kirton (2000) analiza los factores que influyen en la persistencia académica de los estudiantes universitarios de primer curso.

En este sentido, una alternativa que está emergiendo con fuerza es el paradigma de la Resiliencia; partiendo de la idea de que las personas tienen la capacidad de sobreponerse a situaciones difíciles si adquieren competencias específicas para ello (MILSTEIN, 2003). La resiliencia es un constructo que ha recibido muy poca atención en la investigación sobre abandono universitario, pero del que se podrían derivar buenas explicaciones sobre los mecanismos psicológicos que operan en los estudiantes persistentes (LIGHTSEY, 2006).

\section{Resultados.}

En cumplimiento del primer Objetivo se validó y aplicó el cuestionario, del cual se extraen las principales conclusiones, como resultado de la investigación.

Para el cumplimiento de los demás objetivos se triangula la información obtenida con los instrumentos de recogida de datos.

En cumplimiento del objetivo 2 se realizó un comparativo entre la prueba de acceso (saber 11) y los resultados académicos obtenidos en el primer semestre, encontrándose que: 
Para la competencia de lenguaje, el coeficiente de correlación (colegio, universidad) fue $17,15 \%$ lo cual es bajo, por lo que no existe relación directa en el comportamiento de los resultados en las pruebas en la educación media y la educación superior para esta competencia.

Para la competencia de matemática, el coeficiente de correlación (colegio, universidad) fue $27,14 \%$ lo cual también es bajo, por tanto no existe relación directa en el comportamiento de los resultados en las pruebas en la educación media y la educación superior para esta competencia.

En conclusión, el desempeño de las pruebas realizadas es independiente del nivel en que se encuentre el sujeto de estudio, en este caso los estudiantes.

En cumplimiento del objetivo número 3, se analizó la incidencia que tienen los costos educativos, de donde se pudo inferir o que la mayoría deserta por factores económicos incluido en ellos, la consecución de empleo. Este aspecto se confirma con el hecho de que es significativamente bajo el porcentaje de personas que desertan estando becadas. Es necesario resaltar que los estudiantes luego de terminar su nivel tecnológico buscan otras universidades para culminar su ciclo profesional, porque algunas de ellas les homologan mayor número de semestres que las UTS y con ello disminuyen el tiempo y por consiguiente los costos.

En cumplimiento del objetivo 4: se analizó el grado de incidencia que tiene el estrato social, donde se pudieron esta establecer seis componentes: posibilidades económicas, dependencia económica, servicios de salud, ayudas económicas acceso a la tecnología y habilidades financieras.

De estos aspectos se consideran significativos los de posibilidades económicas, dependencia económica, habilidades financieras y ayudas económicas. Poco o nada significativos los servicios de salud y el acceso a la tecnología.

Los estudiantes no tienen dificultad para adquirir los materiales de estudio, ni para transportarse, por consiguiente no son factores que determinen la deserción.

En referencia al aspecto económico, que se perfila como la principal causa de la deserción, los estudiantes consideran que el costo no es alto, comparativamente con otras instituciones, sin embargo para muchos no es posible asumir el valor de la matrícula; además que deben satisfacer en primer lugar sus necesidades básicas como su alimentación, la cual según los encuestados, no es la más adecuada.

Los factores económicos que influyen en la deserción también se ven afectado por el hecho de que más de un $60 \%$ debe hacerse cargo de otra u otras personas además de sus propios gastos. Es decir las obligaciones familiares de manutención priman sobre la decisión de cursar un programa académico. También se evidenció que no existe una tendencia al ahorro, lo cual es indicador de una situación económica limitada y/o poca habilidad para planificar los gastos.

En cumplimiento del objetivo 5: dentro de los aspectos familiares y personales de mayor incidencia se pudieron establecer cinco componentes: habilidades adaptativas; situaciones de conflicto; cambios a nivel familiar, consumo de sustancias y prejuicios limitantes.

Los componentes de esta categoría se muestran como altamente significativos, situación que se enmarca dentro de los fundamentos teóricos del modelo psicoeducativo, donde la resiliencia se concibe como la habilidad fundamental que un estudiante permanezca. Es decir una persona con altas habilidades personales y de relación con otros, tendrá la fortaleza para superar otro tipo de obstáculos que pudieran incentivar la deserción. Así las cosas se muestran como aspectos para fortalecer en aras de disminuir la deserción, las habilidades para adaptarse a nuevas 
situaciones, a las dinámicas de grupo, tolerancia a la frustración, las habilidades de integración y desarrollo personal.

Aspectos como la procedencia (del campo, de la ciudad o de un estrato determinado), sentirse discriminado, tener conflictos familiares, haber sufrido una enfermedad, cambiar de domicilio, cambiar de estado civil, las creencias religiosas, entre otros, solo influyen en la medida en que los factores personales no se encuentren fortalecidos.

En relación con el consumo de sustancias psicoactivas, el consumo de alcohol tiene unos resultados diferentes frente a la proporción de estudiantes consumidores de sustancias psicoactivas. Es preciso denotar que la aceptación social del alcohol hace que no sea percibido como un problema, pero los datos dejan ver que en su gran mayoría los desertores son consumidores de alcohol, lo cual puede convertirse en un factor causal.

Aunque la gran mayoría afirma no haber tenido conflictos con los docentes, es pertinente observar, que es superior el indicie de conflictos con los docentes que con sus propios compañeros.

Dentro de los factores de orden personal, se encuentra la falta de habilidad para hablar en público, las dificultades para adaptarse al grupo o el sentirse discriminado por un docente o un compañero.

Aspectos como el apoyo familiar, la situación social, la ubicación geográfica, el transporte, la edad, el gusto por el programa elegido no resultaron ser aspectos que influyeran en la decisión de desertar.

En cumplimiento del objetivo 6: En el análisis de los factores de orden académico: se encuentra hábitos de estudio inadecuados, la falta de una conducta académica que lo motive hacia la construcción de su propio conocimiento (talleres, foros, revistas, mesas de discusión, etc.); falta de retroalimentación en las evaluaciones; no haber hecho de uso de las tutorías o cursos de nivelación.

Respecto a los programas que conforman la facultad, se tiene que a mayor intensidad de áreas relacionadas con las matemáticas, y mayor la exigencia en procesos de pensamiento, mayor es el índice de deserción.

En la categoría de los "aspectos institucionales" se establecen seis componentes: Metodología y procesos administrativos; Identidad institucional; Procesos académicos; Servicios de Bienestar; Discriminación; Apoyos institucionales.

En esta categoría se muestra más relevante el factor de procesos administrativos, como el cruce de horarios, cancelación de cursos o desconocimiento de algunos procedimientos que afectaron su vida universitaria; así por ejemplo, es significativo que el $24.7 \%$ de estudiantes haya dejado de ver alguna materia por cancelación de cursos; situación que sumada a la dificultad por el cruce de horarios generan una variable que depende de la capacidad de la institución para atender de manera planificada distintas situaciones que afectan la programación académica, dificultando que los estudiantes cursen de manera regular toda la carrera.

\section{Conclusiones}

De los instrumentos aplicados, se obtienen datos que permiten caracterizar la población desertora y en segundo momento se triangula la información para inferir aquellos factores de tipo institucional, social y personal que más influyen en la deserción. 
En la categoría "Actitudinal y Vocacional" el aspecto más significativo fue el de las conductas relacionadas con el programa, donde se establece que en general los estudiantes no tienen una conducta académica que les permita afianzar la vocación por su carrera. Aunque los estudiantes manifiestan gusto por el programa del que desertaron, no tienen una conducta académica (ningún tipo de profundización en algún tópico de la carrera) que sea coherente con tal afirmación.

En la categoría "aspectos socioeconómicos" se puede decir que los estudiantes no tienen dificultad para adquirir los materiales de estudio, y poseen el suficiente dinero para transportarse, por consiguiente no son factores que determinen la deserción. Sin embargo aunque los estudiantes consideran que el costo no es alto, comparativamente con otras instituciones para muchos no es posible asumir el valor de la matrícula; además que deben satisfacer en primer lugar sus necesidades básicas como lo es alimentación.

Por otra parte La mayoría de estudiantes han estado vinculados laboralmente durante su época de estudio, de lo cual se infiere que el desarrollo de estas dos actividades simultáneas puede influir en el fenómeno de la deserción.

En la categoría "aspectos personales y familiares" los datos dejan ver que en su gran mayoría los desertores son consumidores de alcohol u sustancias psicoactivas, lo cual puede convertirse en un factor causal de este fenómeno. En otro aspecto la gran mayoría afirma no haber tenido conflictos con los docentes, pero es pertinente observar, que es superior el indicie de conflictos con los docentes que con sus propios compañeros.

En la categoría "aspectos institucionales" se muestra más relevante el factor de procesos administrativos, cancelación de cursos o desconocimiento de algunos procedimientos que afectaron su vida universitaria situación que sumada a la dificultad por el cruce de horarios generan una variable que depende de la capacidad de la institución para atender de manera planificada distintas situaciones que afectan la programación académica, dificultando que los estudiantes cursen de manera regular toda la carrera.

En la categoría "hábitos y métodos de estudio" se encuentra que los desertores manifiestan haber faltado a clase con alguna regularidad y no haber hecho uso de las tutorías que brinda la institución a pesar de que en muchas ocasiones sienten la necesidad de que se les vuelva a explicar el tema. Se acepta que en mayor o menor proporción, han cambiado con frecuencia sus metas y tiempos de estudio por otras actividades.

Conforme a las categorías estudiadas se recomienda la implementación de una política integral que establezca tareas específicas para cada una de ellas, para lo cual se sugiere la siguiente matriz:

\footnotetext{
CATEGORÍA

Aspectos

actitudinales

ACCIONES SUGERIDAS

vocacionales.

Orientación vocacional a estudiantes de undécimo y aspirantes inscritos.

$\boldsymbol{y}$ Información completa y específica de cada uno de los programas y el perfil de los egresados, incluyendo posibilidades laborales.

Talleres de inducción y superación personal.

Realizar eventos académicos que incentiven el interés y el intercambio de saberes.

Promover la conformación y consolidación de semilleros de investigadores.

Suscripciones a revistas o publicaciones relacionadas con los programas.

Incentivar círculos literarios.

Establecer alianzas que permitan las prácticas o el ejercicio laboral en actividades relacionadas con los programas académicos.

Intercambios académicos con otras instituciones de educación superior.
} 


\section{CATEGORÍA}

Aspectos

Socioeconómicos

\section{ACCIONES SUGERIDAS}

Incentivos a través de becas con un aporte de estudiante para desarrollar su identidad con la institución.

Programas de financiación.

Alianzas con otras entidades que financien los programas académicos (ICETEX, Bancos, Cooperativas, Etc.)

Convenio con empresas del sector para aumentar el mercado laboral de los egresados.

Flexibilidad curricular para que los estudiantes puedan desempeñarse laboralmente de manera simultánea con su programa de estudio.

Apoyo a través de herramientas virtuales.

Capacitaciones sobre habilidades financieras y utilización eficiente del tiempo y de recursos.

Aspectos Servicio de psicología para los estudiantes en temas específicos como:

Personales familiares

Aspectos

Institucionales

y Autoestima

Inteligencia emocional

Resiliencia

Resolución de conflictos

Desarrollo personal.

Talleres y campañas sobre:

Salud sexual y reproductiva.

Prevención del tabaquismo y consumo de sustancias psicoactivas.

Realización de actividades de integración: deportivas, culturales, artísticas etc.

Capacitación a docentes para detectar y prevenir situaciones de conflicto, orientando al estudiante en la búsqueda de los apoyos requeridos.

Formación a los docentes en manejos de grupos y equipos de trabajo.

Flexibilizar los horarios de tal forma que el estudiante pueda desempeñarse laboralmente.

Homogenizar el modelo pedagógico y las estrategias que se derivan del él, de tal forma que sean compartidas por todos los docentes.

Brindar tutorías en diferentes modalidades como apoyo a la autorregulación del aprendizaje.

Planificar tiempos y espacios, de tal forma que no se produzcan traumatismos en los estudiantes por desconocimiento, cambios inesperados o cancelaciones de cursos.

Comunicación con empresas del sector para disminuir el ausentismo de los estudiantes.

Involucrar los docentes en la promoción de las políticas de retención.

Hacer seguimiento a los desertores e implementar políticas de reingreso.

Programas de inducción a docentes nuevos.

-Campañas de identidad y adhesión institucional.

-Promoción y divulgación del servicio de las tutorías.

El adecuado manejo de relaciones interpersonales como uno de los criterios para la selección de docentes.

Aspectos Hábitos y Utilizar como insumo de los planes de mejora, los resultados de la prueba métodos de diagnóstica.

estudio. Formación docente en didácticas específicas y utilización de organizadores de información.

Hacer seguimiento al ausentismo.

Hacer seguimiento a las tutorías.

Para atender los requerimientos de esta matriz, es preciso que la institución cuente con distintas dependencias que puedan brindar apoyo en diferentes áreas: 
Psicología, Bienestar institucional, Investigación, biblioteca, apoyo financiero, coordinaciones, gestión universitaria, apoyo académico, promoción de la salud, gestión administrativa, recursos humanos, tutorías, alianzas estratégicas, entre otros.

\section{REFERENCIAS BIBLIOGRÁFICAS}

Amaya, G. S., Salcedo, W. N., \& Valencia, A. D. G. (2016). Factores de deserción estudiantil en la universidad surcolombiana. Revista Paideia Surcolombiana, (14), 97-103.

Ángel, Z. M. (2002). La enseñanza universitaria. el escenario y sus protagonistas. Madrid: Narcea,

Bean, J. P., \& Metzner, B. S. (1985). A conceptual model of nontraditional undergraduate student attrition. Review of Educational Research, 55(4), 485-540.

Bourdieu, P., \& Passeron, J. (1990). Reproduction in education, society and culture Sage.

Cabrera, A. F., Nora, A., \& Castaneda, M. B. (1992). The role of finances in the persistence process: A structural model. Research in Higher Education, 33(5), $571-593$.

Cabrera, A. F., Nora, A., \& Castaneda, M. B. (1993). College persistence: Structural equations modeling test of an integrated model of student retention. Journal of Higher Education, , 123-139.

Cabrera, L., Bethencourt, J. T., González, M., \& Álvarez, P. (2006). Un estudio transversal retrospectivo sobre prolongación y abandono de estudios universitarios. Relieve, 12(1), 105-127. 
Campo-Arias, A., \& Oviedo, H. C. (2008). Propiedades psicométricas de una escala: La consistencia interna. Rev Salud Pública, 10(5), 831-839.

Castaño, E., Gallón, S., \& Vásquez, J. (2008). Análisis de los factores asociados a la deserción estudiantil en la educación superior: Un estudio de caso. Revista De Educación, (345), 255-280.

de Escobar, V., de Tirado, N. D., de Santana, O. F., \& Hernández, D. (2005). Estudio sobre la deserción y repitencia en la educación superior en panamá Consejo de Rectores.

DE, RED IBEROAMERICANA PARA LA ACREDITACIÓN. (2004). Glosario internacional riaces de evaluación de la calidad y acreditación.

Giovagnoli, P. I. (2001). Determinantes De La Deserción y Graduación Universitaria,

Girola, L. (2005). Anomia e individualismo: Del diagnóstico de la modernidad de durkheim al pensamiento contemporáneo Anthropos Editorial.

Henderson, N., Milstein, M. M., \& Vitale, G. (2003). Resiliencia en la escuela Paidós Buenos Aires.

Holland, J. L. (1966). The psychology of vocational choice: A theory of personality types and model environments.

Ion, G. (2014). Understanding the role of organizational factors in shaping the research careers of women academics in higher education. Journal of New Approaches in Educational Research, 3(2), 59.

Kirton, M. J. (2003). Transitional factors influencing the academic persistence of first-semester undergraduate freshmen UMI. 
Lewis, L. S., Pascarella, E. T., \& Terenzini, P. T. (1992). How College Affects Students: Findings and Insights from Twenty Years of Research,

Lightsey, O. R. (2006). Resilience, meaning, and well-being. The Counseling Psychologist, 34(1), 96-107.

Lujan, J. R., \& Resendiz, A. (1981). Hacia la construcción de un modelo causal en el análisis de la deserción. México DF: Universidad Autónoma Metropolitana Iztapalapa,

Manrique, J. M. Á. (1997). Etiología de un sueño o el abandono de la universidad por parte de los estudiantes por factores no académicos Universidad Autónoma de Colombia, Sistema Universitario de Investigaciones.

Nora, A. (2001). The depiction of significant others in tinto's "Rites of passage": A reconceptualization of the influence of family and community in the persistence process. Journal of College Student Retention: Research, Theory \& Practice, $3(1), 41-56$.

Nora, A., Cabrera, A., Hagedorn, L. S., \& Pascarella, E. (1996). Differential impacts of academic and social experiences on college-related behavioral outcomes across different ethnic and gender groups at four-year institutions. Research in Higher Education, 37(4), 427-451.

Ortiz, Tomás Sola Martínez Antonio Moreno. (2005). La acción tutorial en el contexto del espacio europeo de educación superior. Educación y Educadores, 8, 123-143.

Pardo, A., \& Ruíz, M. (2002). SPSS. guía para el análisis de datos. Editorial McGrawHill Intera-Mericana Madrid, España, 
Romo, A. (2007). Retención y deserción en un grupo de instituciones mexicanas de educación superior.

Ryan, M. P., \& Glenn, P. A. (2002). Increasing one-year retention rates by focusing on academic competence: An empirical odyssey. Journal of College Student Retention: Research, Theory \& Practice, 4(3), 297-324.

Seligman Martin, E. (1998). Learned optimism.

Thomas, L. (2002). Student retention in higher education: The role of institutional habitus. Journal of Education Policy, 17(4), 423-442.

Tinto, V. (1975). Dropout from higher education: A theoretical synthesis of recent research. Review of Educational Research, 45(1), 89-125.

Tinto, V. (1982). Limits of theory and practice in student attrition. The Journal of Higher Education, , 687-700.

Tinto, V. (1987). Leaving college: Rethinking the causes and cures of student attrition. ERIC.

Tinto, V. (1989). Definir la deserción: Una cuestión de perspectiva. Revista De Educación Superior, 71, 33-51.

Wasserman, K. N. (2001). Psychological and developmental differences between students who withdraw from college for personal-psychological reasons and continuing students

Recebido para publicação em 02-09-16; aceito em 03-10-16 